\title{
Intravenous iron treatment in pregnancy: comparison of high-dose ferric carboxymaltose vs. iron sucrose*
}

\author{
Patricia Christoph ${ }^{1}$, Christine Schuller ${ }^{1}$, \\ Hanna Studer ${ }^{1}$, Olivier Irion ${ }^{2}$, Begoña Martinez \\ De Tejada $^{2}$ and Daniel Surbek ${ }^{1, * *}$ \\ ${ }^{1}$ Department of Obstetrics and Gynecology, University \\ Hospital Insel and University of Bern, Bern, Switzerland \\ ${ }^{2}$ Department of Obstetrics and Gynecology, University \\ Hospital, Geneva, Switzerland
}

\begin{abstract}
Objective: Oral iron substitution has shown to be insufficient for treatment of severe iron deficiency anemia in pregnancy. Ferric carboxymaltose is a new intravenous (i.v.) iron formulation promising to be more effective and as safe as iron sucrose. We aimed to assess side effects and tolerance of ferric carboxymaltose compared to i.v. iron sucrose in pregnant women.

Methods: We performed a retrospective analysis of 206 pregnant women who were treated either with ferric carboxymaltose or iron sucrose for iron-deficiency anemia with intolerability to oral iron substitution, or insufficient hemoglobin increase after oral iron treatment, or need for rapid hemoglobin reconstitution. Primary endpoint was to evaluate the maternal safety and tolerability. Secondary endpoint was to assess efficacy of the treatment and exclude safety concerns for the fetus.

Results: The incidence of drug-related adverse events was low and mostly mild in both groups. Mild adverse events occurred in $7.8 \%$ for ferric carboxymaltose and in $10.7 \%$ for iron sucrose. The mean rise of hemoglobin value was $15.4 \mathrm{~g} / \mathrm{L}$ for ferric carboxymaltose and $11.7 \mathrm{~g} / \mathrm{L}$ for iron sucrose.

Conclusion: Ferric carboxymaltose administration in pregnant women is well tolerated and is not associated with any relevant clinical safety concerns. Ferric carboxymaltose has a comparable safety profile to iron sucrose but offers the advantage of a much higher iron dosage at a time reducing the need for repeated applications and increasing patients' comfort. Ferric carboxymaltose is the drug of choice, if i.v. iron treatment becomes necessary in the second or third trimester of pregnancy.
\end{abstract}

*This study was supported by an unrestricted grant from Vifor Pharma, Switzerland.

**Corresponding author:

Professor Dr. Daniel Surbek

Department of Obstetrics and Gynecology

University Hospital Insel and University of Bern

Effingerstr. 102

3010 Bern

Switzerland

E-mail: daniel.surbek@insel.ch
Keywords: Anemia; ferric carboxymaltose; hemoglobin; intravenous iron therapy; iron deficiency; iron sucrose; pregnancy; safety; tolerability.

\section{Introduction}

Anemia in pregnancy is one of the most frequent problems in Obstetrics. The most common cause of anemia in pregnancy is iron deficiency. Iron-deficiency anemia is a serious public health problem with significant impact on physical development $[23,24]$. Iron deficiency in pregnancy has been defined as low ferritin levels [9], and it is considered the gold standard for the diagnosis of iron-deficiency anemia in pregnancy, together with hemoglobin values [19].

The clinical manifestations of anemia include skin or mucosal pallor, lack of energy and shortness of breath, fatigue, lack of concentration and decreased mental, physical, and cognitive performance all of which can present in different grades depending on the severity of the anemia [2]. Consequences of moderate to severe anemia in pregnancy are susceptibility to infection and premature delivery, intrauterine growth restriction, and the consequences of prematurity: increased perinatal morbidity and mortality $[13,21]$. This also means that anemia at the time of the delivery results more often in the need for blood transfusion, increased cardiovascular risks, longer hospital stay, and problems in the postpartum period like reduced lactation or postpartum mood disorder [7]. For the neonate, there is a risk of reduced iron stores with serious consequences for their development [11, $12,21]$.

There are various possible forms of treatment for irondeficiency anemia. Oral iron is the preferred route of administration for mild anemia. Treatment with iron preparations is used routinely in pregnancy, if iron deficiency with or without anemia develops. However, oral iron supplementation often leads to adverse effects, such as constipation, abdominal pain, or sickness. If these unwarranted gastrointestinal effects arise, adherence to iron treatment decreases.

Intravenous (i.v.) iron preparations are promising, especially in cases of severe anemia. They provide a greater and more rapid iron supply than oral iron therapy without the gastrointestinal side effects of oral substitution and make it possible to avoid blood transfusion with associated risks [16]. To date, few studies have focused on the use of i.v. iron and its side effects and safety in pregnant women. Iron sucrose has been used for years for i.v. treatment of iron deficiency in pregnant women after the first trimester. However, its use is limited to low dose due to local and systemic side effects in higher doses. Recently, ferric carboxymaltose has been introduced. This iron 
preparation can be used intravenously in high doses with up to $1000 \mathrm{mg}$ infused in $15 \mathrm{~min}$ and low risk of side effects. We have previously shown that ferric carboxymaltose does not cross the placental barrier in an in vitro dual perfusion model (15), and its use is approved in the second and third trimesters of pregnancy. However, no published data are available concerning the clinical use of ferric carboxymaltose use in pregnant women. The aim of our study was, therefore, to compare i.v. ferric carboxymaltose with i.v. iron sucrose during pregnancy regarding the tolerability and safety profile.

\section{Methods}

We performed a retrospective observational study to analyze the tolerability and efficacy of i.v. iron therapy in pregnancy. Data were obtained from the maternity units in the University Hospitals of Berne and Geneva in Switzerland. The study was approved by the local ethics committees of the respective institutions.

All pregnant women who received i.v. ferric carboxymaltose since the approval of this new drug in Switzerland in February 2008 were eligible for entry into the study. The comparison group was formed by a group of equal number of pregnant women who were treated with i.v. iron sucrose, before ferric carboxymaltose was introduced on the market (i.e., before February 2008).

Laboratory testing, indication, and prescription of the iron substitution treatment were determined by the attending medical team. Inclusion criteria for i.v. iron treatment at both centers were according to specific local maternity guidelines on diagnosis and treatment of anemia in pregnancy as well as to specific national guidance [7]. These included the following: pregnancy, clinically relevant severe iron-deficiency anemia, intolerance to oral iron substitution, failure of hemoglobin increase after oral iron treatment, or need for rapid hemoglobin- reconstitution. Patients with early pregnancy (before 13 weeks gestation) were excluded.

Patients were identified by searching digital records of the hospitals. Baseline data were collected on maternal age and weight, gestational age, results from peripheral blood counts before and after treatment, serum ferritin prior to treatment, adverse events during i.v. iron treatment, and pregnancy outcomes. i.v. iron was administered by nurses or midwives, and they were instructed to document the procedure and any side effects during and after i.v. administration.

The primary endpoint of this study was to evaluate the maternal tolerability and side effects of ferric carboxymaltose compared to iron sucrose being used for treatment of iron-deficiency anemia in the second and third trimesters of pregnancy. Secondary endpoints included efficacy of the treatment and signs for concern regarding the fetal safety. For the efficacy analysis, only women with ferritin levels $\leq 30 \mu \mathrm{g} / \mathrm{L}$ were included as a ferritin level $\leq 30 \mu \mathrm{g} / \mathrm{L}$ is required for the diagnosis of iron-deficiency anemia.

Statistical analysis was performed using Windows Excel Calculation and Graphpad InStat (GraphPad Software, Inc., La Jolla, CA, USA). Continuous variables were compared using Student's $t$ test; categorical variables were compared using $\chi^{2}$-test or Fisher's exact test where applicable. For all analyses, P-values $<0.05$ were considered statistically significant.

\section{Results}

A total of 206 pregnant women were included in the study; 103 received ferric carboxymaltose since February 2008, and 103 received iron sucrose between 2005 and 2007. Demographic characteristics and basic data are summarized in Table 1. While demographic data did not show any significant difference between groups, there was a statistically highly significant difference regarding the administered iron dose and the repeated application. Patients in the ferric carboxymaltose group received, in average, the double dose of iron weekly (Table 1). More patients in the iron sucrose group received repetitive doses of iron intravenously. These differences correspond to the recommended treatment schemes of ferric carboxymaltose and iron sucrose, respectively; ferric carboxymaltose can be administered in much higher single doses than iron sucrose.

\section{Side effects, tolerance}

As summarized in Table 2, patients treated with ferric carboxymaltose had fewer side effects than those receiving iron sucrose, but the difference did not reach statistical significance. Mild local reactions at the injection site were reported by three patients after receiving ferric carboxymaltose. One reported local pain and swelling at the injection site, the two other patients reported a rush on the arms and legs. Mild systemic reactions were reported by five patients, three patients had a transient hypotension (systolic blood pressure $<100$ $\mathrm{mm} \mathrm{Hg}$ ), one of them also described dizziness, and another one had headache. Concerning the hypotension, no medical intervention was required, and their blood pressures normalized spontaneously. In the group receiving iron sucrose, a total of 11 adverse events were reported, 8 mild systemic reactions and 3 local reactions. The systemic reactions consisted in five cases of mild hypotension with dizziness and headache, two cases of heart palpitation during/after the infusion, and one case of nausea. Figure 1 graphically depicts the differences between groups.

\section{Fetal safety and neonatal outcome}

Among women treated with ferric carboxymaltose, $60.2 \%$ were hospitalized due to high-risk pregnancy, such as preterm labor, intrauterine growth restriction, or abnormal placentation. They were closely monitored with daily CTG, Doppler ultrasound twice a week, and biophysical profile every 2 weeks. No signs of negative effects of the i.v. iron treatment were detected on the fetus.

Of all women treated with ferric carboxymaltose, $77 \%$ delivered healthy babies at term. Twenty-three percent of the women delivered preterm babies due to complications, which were present before ferric carboxymaltose administration, related to their high-risk pregnancies.

Among the women treated with i.v. iron sucrose, fewer had high-risk pregnancies, and fewer had inpatient treatment. This explains the difference in neonatal outcome: in this group, 91 , $8 \%$ delivered healthy babies at term, $8,2 \%$ had preterm delivery, all of them due to high-risk pregnancy situations other than anemia. Among the women treated with i.v. iron sucrose, $16.5 \%$ had been treated as inpatients due to their high-risk situation. 
Table 1 Demographic characteristics and base data.

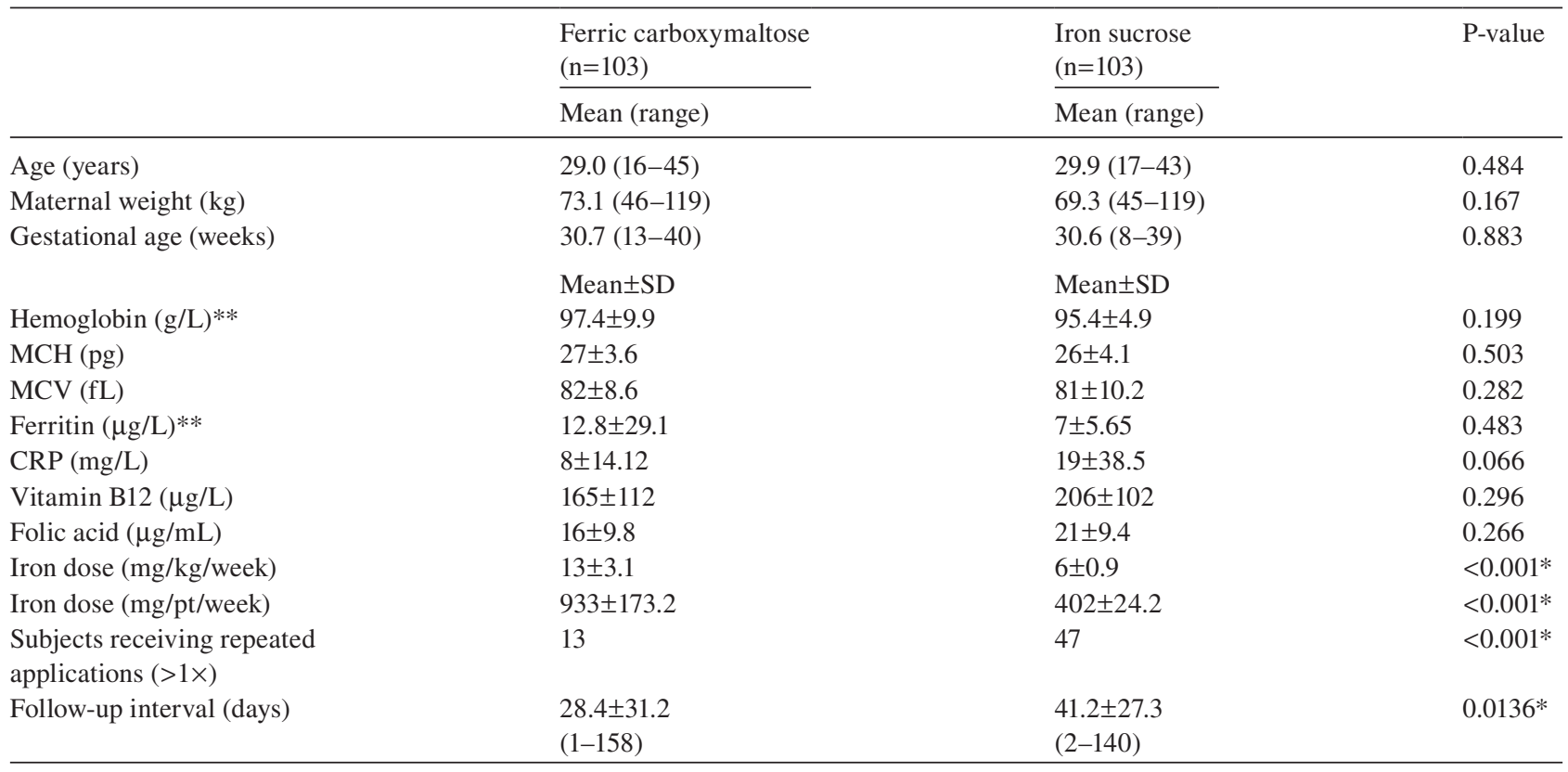

*Statistically significant.

**Value prior to first drug administration.

The gestational age at birth between both groups was statistically different: ferric carboxymaltose group (38.4 \pm 3.26$)$ vs. iron sucrose group $(39.6 \pm 2.65), P=0.009$. Therefore, more babies in the ferric carboxymaltose group were transferred to NICU due to prematurity. There were no differences between both groups in the APGAR scores and umbilical cord $\mathrm{pH}$ values. There was no statistically significant difference between both groups regarding intrauterine deaths and neonatal deaths.

\section{Efficacy of i.v. iron treatment}

In the group treated with ferric carboxymaltose, $85 \%$ had a ferritin level $\leq 30 \mu \mathrm{g} / \mathrm{L}$. In the group treated with iron sucrose, $77 \%$ had a ferritin level $\leq 30 \mu \mathrm{g} / \mathrm{L}$. Table 3 shows mean hemoglobin values in both groups before and after i.v. iron treatment. Women treated with iron sucrose received $400 \mathrm{mg}$ iron per week in two infusions, $48 \mathrm{~h}$ apart. Fortyseven patients received repeated applications up to eight times. The maximum dose of $500 \mathrm{mg} /$ week was never exceeded. For the mean, every woman treated with iron sucrose was given $6 \mathrm{mg}$ iron sucrose $/ \mathrm{kg}$ body weight per week (Table 1).

Table 2 Local and systemic adverse events.

\begin{tabular}{|c|c|c|c|}
\hline & \multirow{2}{*}{$\frac{\text { Ferric carboxymaltose }}{(n=103)}$} & \multirow{2}{*}{$\frac{\text { Iron sucrose }}{(\mathrm{n}=103)}$} & \multirow{2}{*}{ P-value } \\
\hline & & & \\
\hline Local reactions & $3(2.9 \%)$ & $3(2.9 \%)$ & 1.000 \\
\hline Systemic reactions & $5(4.9 \%)$ & $8(7.8 \%)$ & 0.568 \\
\hline Adverse events total & $8(7.8 \%)$ & $11(10.7 \%)$ & 0.631 \\
\hline
\end{tabular}

Women treated with ferric carboxymaltose received up to $1300 \mathrm{mg}$ iron per week. On average, $13 \mathrm{mg} / \mathrm{kg}$ body weight per week was given. This corresponds to more than double the amount of iron that was administered with iron sucrose. Only 13 patients had one more repetitive application. Most patients just got $1000 \mathrm{mg}$ ferric carboxymaltose i.v. In two women with body weights below $60 \mathrm{~kg}$, the recommended maximal ferric carboxymaltose dose of $15 \mathrm{mg} / \mathrm{kg}$ body weight was exceeded with no associated noticeable negative effect.

The mean hemoglobin rise in the group receiving ferric carboxymaltose was $15.4 \mathrm{~g} / \mathrm{L}$ and $11.7 \mathrm{~g} / \mathrm{L}$ in the group receiving iron sucrose. The mean follow-up time was different in both groups after i.v. iron administration. The mean follow-up for the group treated with ferric carboxymaltose was 28.4 days and 41.2 days for the group treated with iron sucrose. This difference is statistically significant.

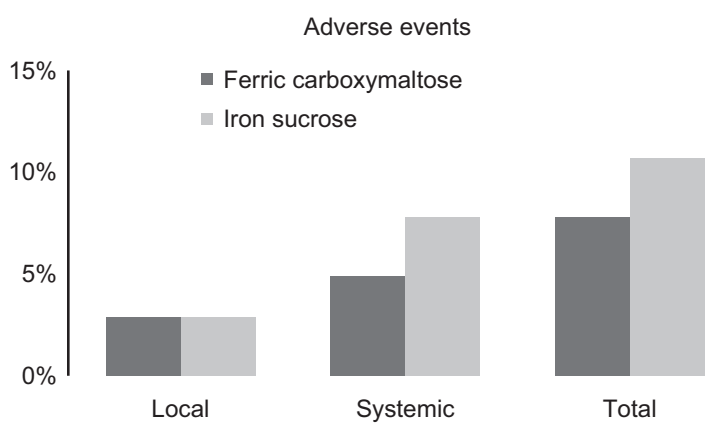

Figure 1 Local and systemic adverse events. 
Table 3 Hemoglobin values before and after treatment.

\begin{tabular}{|c|c|c|c|}
\hline & \multicolumn{2}{|l|}{ Mean \pm SD range } & \multirow[t]{2}{*}{ P-value } \\
\hline & $\begin{array}{l}\text { Ferric } \\
\text { carboxymaltose }\end{array}$ & $\begin{array}{l}\text { Iron } \\
\text { sucrose }\end{array}$ & \\
\hline $\begin{array}{l}\text { Hemoglobin before } \\
\text { treatment }(\mathrm{g} / \mathrm{L})\end{array}$ & $\begin{array}{l}98.3 \pm 10.9 \\
77-125\end{array}$ & $\begin{array}{l}95.6 \pm 12.1 \\
71-140\end{array}$ & 0.1581 \\
\hline $\begin{array}{l}\text { Hemoglobin after } \\
\text { treatment }(\mathrm{g} / \mathrm{L})\end{array}$ & $\begin{array}{l}113.1 \pm 12.3 \\
81-149\end{array}$ & $\begin{array}{l}110.4 \pm 11.9 \\
88-147\end{array}$ & 0.2603 \\
\hline $\begin{array}{l}\text { Rise in hemoglobin } \\
\text { value }(\mathrm{g} / \mathrm{L})\end{array}$ & $\begin{array}{l}15.4 \pm 11.7 \\
-5 \text { to } 43\end{array}$ & $\begin{array}{l}11.7 \pm 10.0 \\
-4 \text { to } 43\end{array}$ & 0.0819 \\
\hline
\end{tabular}

\section{Discussion}

Screening for iron-deficiency anemia in pregnancy has been based on the association with increased risk of preterm delivery and low birth weight, and maternal signs and symptoms of anemia. While the consequences of mild to moderate irondeficiency anemia for the fetus may be overestimated [2], severe iron-deficiency anemia leads to increase in morbidity of pregnant women and their children in developing as well as in developed countries.

In the majority of cases, anemia can be treated effectively with oral iron preparations. Many patients tolerate oral intake of iron supplements well; however, up to $40 \%$ have side effects related to oral iron treatment. The incidence of adverse reactions is dose dependent. The main adverse effects are of gastrointestinal nature [1, 5, 7], the most common being constipation, diarrhea, epigastric discomfort, nausea, severe abdominal pain, or vomiting. These secondary effects can be lessened by the intake of tablets after meals, although this leads to a concomitant reduction of iron absorption. Typically, these adverse effects lead to poor treatment adherence especially in pregnancy when similar gastrointestinal complaints are often a problem prior to iron treatment due to the physiological changes in pregnancy. Intolerance to oral iron intake leads to a greater percentage of failure in the treatment. In addition, even with strict oral iron treatment adherence, there are still quite a number of patients who do not respond with an appropriate hemoglobin increase (i.e., hemoglobin increase below $10 \mathrm{~g} / \mathrm{L}$ within 14 days) [17].

Other possible indications for i.v. iron treatment include the necessity of rapid increase of hemoglobin, for example, pregnancies in the third trimester with high-risk of peripartum hemorrhage (e.g., placenta previa or placenta increta), or Jehovah's Witnesses [21].

In these clinical situations described above, i.v. iron administration is indicated. A faster increase in hemoglobin, ferritin, and iron stores by i.v. iron therapy has been reported by different authors $[5,6,8,10,15,17,20-22]$.

Severe anemia mainly occurs in developing countries where it has been attributed to poor nutrition and concurrent conditions, especially infectious diseases, such as malaria. These women particularly benefit from high-dose i.v. iron substitution because availability of blood transfusions is very limited, and blood transfusions still bare certain risks. I.V. iron substitution in this low-resource setting seems to be a very good and safe alternative.

\section{Side effects and tolerance}

The tolerance and efficacy of ferric carboxymaltose has been demonstrated previously in several studies for different groups of patients with iron-deficiency anemia $[3,4,7$, $8,14,19,21]$ with similar results. Bailie GR [3] showed in a review paper, including nine randomized studies with more than 3000 patients, that ferric carboxymaltose had a good tolerability and efficiency profile. The use of ferric carboxymaltose for treatment of postpartum anemia has been extensively investigated $[7,19,21]$. No safety concerns have been identified in breastfed infants of mothers receiving ferric carboxymaltose [7].

Ferric carboxymaltose is approved for use in pregnancy in the second and third trimesters. However, up to now, no published data from clinical studies investigating the use of ferric carboxymaltose in pregnancy are available.

Our study shows that ferric carboxymaltose is well tolerated in pregnant women and has fewer or equal number of side effects compared to the previously used iron sucrose when administered in a dose double as high. The incidence of drug-related adverse events was low and comparable to those described for ferric carboxymaltose and iron sucrose in other studies. Registered adverse events were all classified as mild and quickly reversible and mostly restricted to local reactions at the infusion site. There were no treatment-related serious adverse events. No anaphylactic or anaphylactoid reaction was detected. No venous thrombosis was registered. None of the adverse events required further medical intervention.

\section{Fetal safety and neonatal outcome}

Previously, an in vitro study using a dual-placenta perfusion model has shown that ferric carboxymaltose does not cross the placental barrier to the fetal side [15]. Though there is no previous published clinical data available on the use of ferric carboxymaltose in pregnancy and its effects on the fetus, ferric carboxymaltose is approved for use in the second and third trimesters of pregnancy. We, therefore, have chosen to give ferric carboxymaltose initially in an inpatient setting to be able to closely monitor the pregnant women and the fetus for adverse reactions and negative effects. The pregnant women being treated as inpatients were mainly high-risk pregnancies due to other complications than IDA. This explains why the gestational age was significantly higher in the iron sucrose group compared to the group receiving ferric carboxymaltose, and therefore, birth weight at delivery was also higher in the group of iron sucrose. Newborn intensive care unit (NICU) admission rate was higher in the group of newborns from women receiving ferric carboxymaltose due to a lower gestational age at delivery in this group with no correlation to i.v. iron treatment. There was no statistically significant difference between both groups regarding intrauterine deaths and neonatal deaths. Both groups of pregnant women were heterogeneous, and therefore not really comparable, regarding 
pregnancy complications. Nevertheless, no sign for negative effect on the fetus of iron infusion could be detected, although levels of ferritin were not determined in newborns.

\section{Efficacy}

I.V. iron treatment indications were heterogeneous and did not always correspond to national recommendations. Owing to heterogeneity in indications for i.v. iron treatment and in dosage of the drugs and also regarding follow-up intervals in our study population, comparability of efficacy of ferric carboxymaltose vs. iron sucrose is limited. Both i.v. iron preparations are effective in treating anemia in pregnancy. The differences between the administered amounts of iron were statistically significant when giving ferric carboxymaltose and iron sucrose as well as the repetitions of the treatment (Table 1). These facts suggest that the larger the amount of iron that can be administered in the form of ferric carboxymaltose, the more effective the replenishment of the iron stores is and the more effective the correction of the anemia is. This, however, needs to be confirmed in appropriately designed prospective studies.

Whether ferric carboxymaltose provides a faster and higher increase in hemoglobin level than iron sucrose cannot be concluded from these data due to the possibility of administering a higher dose of iron within a shorter time period.

\section{Strength of the study}

This is the first study assessing the prevalence of side effects and tolerability of i.v. ferric carboxymaltose treatment in pregnant women. Data on unwarranted side effects are reliable as they are prospectively collected and documented during and after treatment. Ferric carboxymaltose was used mainly in an inpatient population to ensure maximal safety, observation, and monitoring.

\section{Limitations of the study}

The retrospective design of the study determines the limitations. Indications for i.v. iron treatment, dosages of ferric carboxymaltose and iron sucrose varied according to clinical situations. The groups were different before i.v. iron treatment regarding pregnancy complications, limiting interpretation of neonatal outcome data. Follow-up data to evaluate outcome was inhomogenic, and no data were available on ferritin and transferrin saturation after the treatment. Regarding the neonatal outcome, no data on the hematological status and ferritin levels were available. Neither the impact of anemia on maternal quality of life nor the benefits of the treatment were assessed.

In summary, our study shows that the tolerance of ferric carboxymaltose in pregnancy is excellent, and prevalence of side effects is low, even when administered in a much higher iron dose compared to iron sucrose. Compared to iron sucrose, it offers the advantage of a much higher iron dosage at a time reducing the need for repeated applications and increasing patients' comfort. No relevant clinical safety concern of ferric carboxymaltose regarding either mother or fetus could be identified at short and midterm.

In view of the limited evidence from prospective randomized trials of i.v. iron treatment in pregnancy, full consideration must be given to possible benefits and risks. Indications for the use of parenteral iron should be limited to specific conditions, in which oral iron supplementation is not possible or fails.

While further research including randomized trials is needed [18], ferric carboxymaltose seems to be the drug of choice if i.v. iron treatment during pregnancy becomes necessary in the second or third trimester.

\section{References}

[1] Al RA, Unlubilgin E, Kandemir O, Yalvac S, Cakir L, Haberal A. Intravenous versus oral iron for treatment of anemia in pregnancy: a randomized trial. Obstet Gynaecol. 2005;106: 1335-40.

[2] Al-Momen AK, Al-Meshari A, Al-Nuaim L, Saddique A, Abotalib Z, Khashogji $\mathrm{T}$, et al. Intravenous iron sucrose complex in the treatment of iron deficiency anemia during pregnancy. Eur J Obstet Gynecol Reprod Biol. 1996;69:121-4.

[3] Bailie GR. Efficacy and safety of ferric carboxymaltose in correcting iron-deficiency anemia: a review of randomized controlled trials across different indications. Arzneimittelforschung. 2010;60:386-98.

[4] Bashiri A, Burstein E, Sheiner E, Mazor M. Anemia during pregnancy and treatment with intravenous iron: review of the literature. Eur J Obstet Gynecol Reprod Biol. 2003;110:2-7.

[5] Bayoumeu F, Subiran-Buisset C, Baka NE, Legagneur H, Monnier-Barbarino $\mathrm{P}$, Laxenaire $\mathrm{MC}$. Iron therapy in iron deficiency anemia in pregnancy: intravenous route versus oral route. Am J Obstet Gynecol. 2002;186:518-22.

[6] Bencaiova G, von Mandach U, Zimmermann R. Iron prophylaxis in pregnancy: intravenous route versus oral route. Eur J Obstet Gynecol Reprod Biol. 2009;144:135-9.

[7] Breymann C, Gliga F, Bejenariu C, Strizhova N. Comparative efficacy and safety of intravenous ferric carboxymaltose in the treatment of postpartum iron deficiency anemia. Int J Gynaecol Obstet. 2008;101:67-73.

[8] Breymann C, Honegger C, Holzgreve W, Surbek D. Diagnosis and treatment of iron-deficiency anaemia during pregnancy and postpartum. Arch Gynecol Obstet. 2010;282:577-80.

[9] Institute of Medicine, Committee on Nutritional Status During Pregnancy and Lactation. Nutrition during pregnancy. Washington, DC: National Academy Press; 1990. p. 272-98.

[10] Khalafallah A, Dennis A, Bates J, Bates G, Robertson IK, Smith $\mathrm{L}$, et al. A prospective randomized, controlled trial of intravenous versus oral iron for moderate iron deficiency anaemia of pregnancy. J Intern Med. 2010;268:286-95.

[11] Klebanoff MA, Shiono PH, Selby JV, Trachtenberg AI, Graubard BI. Anemia and spontaneous preterm birth. Am J Obstet Gynecol. 1991;164:59-63.

[12] Liberman E, Ryan KJ, Monson RR, Schooenbaum SC. Association of maternal hematocrit with premature labor. Am J Obstet Gynecol. 1988;139:107-14.

[13] Lone FW, Qureshi RN, Emmanuel F. Maternal anemia and its impact on perinatal outcome in a tertiary care hospital in Pakistan. Eastern Mediterr Health J. 2004;10:801-7. 
[14] Lyseng-Williamson KA, Keating GM. Ferric carboxymaltose: a review of its use in iron-deficiency anaemia. Drugs. 2009;69:739-56.

[15] Malek A. In vitro studies of ferric carboxymaltose on placental permeability using the dual perfusion model of human placenta. Arzneimittelforschung. 2010;60:354-61.

[16] Milman N. Prepartum anaemia: prevention and treatment. Ann Hematol. 2008;87:949-59.

[17] Puolakka J, Janne O, Vihko R. Serum ferritin in the diagnosis of anemia during pregnancy. Acta Obstet Gynecol Scand. 1980;95(Suppl):57-63.

[18] Reveiz L, Gyte GML, Cuervo LG. Treatments for irondeficiency anaemia in pregnancy. Cochrane Database Syst Rev. 2007;18:CD003094. doi:10.1002/14651858.CD003094.pub2.

[19] Seid MH, Derman RJ, Baker JB, Banach W, Goldberg C, Rogers $R$. Ferric carboxymaltose injection in the treatment of postpartum iron deficiency anemia: a randomized controlled clinical trial. Am J Obstet Gynecol. 2008;199:435.e1-e7.

[20] Singh K, Fong YF, Kuperan P. A comparison between intravenous iron polymatose complex (ferrum Hausmann) and oral ferrous fumarate in the treatment of iron deficiency anaemia in pregnancy. Eur J Haematol. 1998;60:119-24.

[21] Van Wyck DB, Martens MG, Seid MH, Baker JB, Mangione A. Intravenous ferric carboxymaltose compared with oral iron in the treatment of postpartum anemia: a randomized controlled trial. Obstet Gynecol. 2007;110:267-78.

[22] Worldwide prevalence of anaemia 1993-2005: WHO global database on anaemia. Edited by Bruno de Benoist, Erin McLean, Ines Egli and Mary Cogswell.

[23] World Health Organization. Nutritional anaemias. Tech Rep Ser. 1972;503.

[24] World Health Organization. Preventing and controlling iron deficiency anemia through primary healthcare. Geneva; WHO 1989.

The authors stated that there are no conflicts of interest regarding the publication of this article.

Received September 15, 2011. Revised February 29, 2012. Accepted March 27, 2012. Previously published online May 13, 2012. 